\title{
Universiteit
}

Leiden

The Netherlands

\section{Honey in traditional Chinese medicine: A guide to future applications of NADES to medicines}

Dai, Y.; Choi, Y.H.; Verpoorte, R.

\section{Citation}

Dai, Y., Choi, Y. H., \& Verpoorte, R. (2021). Honey in traditional Chinese medicine: A guide to future applications of NADES to medicines. Advances In Botanical Research, 97, 361-384. doi:10.1016/bs.abr.2020.09.011

Version: Publisher's Version

License: $\quad$ Licensed under Article 25fa Copyright Act/Law (Amendment Taverne)

Downloaded from: $\quad$ https://hdl.handle.net/1887/3248726

Note: To cite this publication please use the final published version (if applicable). 


\title{
Honey in traditional Chinese medicine: A guide to future applications of NADES to medicines
}

\author{
Yuntao Dai ${ }^{\mathrm{a}, *}$, Young Hae Choi ${ }^{\mathrm{b}}$, and Robert Verpoorte ${ }^{\mathrm{b}}$ \\ ${ }^{a}$ Institute of Chinese Materia Medica, China Academy of Chinese Medical Sciences, Beijing, China \\ ${ }^{\mathrm{b}}$ Natural Products Laboratory, Institute of Biology, Leiden University, Leiden, The Netherlands \\ *Corresponding author: e-mail address: dai_yuntao@live.cn
}

\section{Contents}

1. Honeybee products, their ingredients and traditional applications 362

1.1 Traditional applications of honey 362

1.2 Chemical ingredients of honey 365

1.3 Honey as a NADES? 366

2. Honey in traditional Chinese medicine 368

2.1 Honey stir-fried preparations 369

2.2 Traditional formulations: Honeyed pills and other dosage forms 375

3. The physico-chemical properties of honey as NADES 378

$\begin{array}{ll}3.1 \text { Hygroscopicity } & 378\end{array}$

$\begin{array}{ll}3.2 \text { Viscosity } & 378\end{array}$

$\begin{array}{ll}3.3 \text { Refractive index } & 378\end{array}$

$\begin{array}{ll}3.4 & \text { Optical rotation } \\ & 378\end{array}$

4. Other adjuvants in TCM 380

5. Prospects 381

References 382

\begin{abstract}
Honey has played an important role in traditional medicine for thousands of years. It has been used in different aspects of traditional medicine, as a single medicine, as an additive for paozhi processing, and as an adjuvant for different types of pharmaceutical formulations, such as electuary, honeyed pills, etc. Hundreds of compounds have already been found in honeys from different sources. However, the major compounds in honey are glucose and fructose in about equal molar ratios. This means that the basis of honey is a natural deep eutectic solvent (NADES). The physico-chemical properties of NADES may play an important role in the use of honey in traditional medicine. Clarifying the role of honey in traditional medicines helps to rationalize traditional practices, as shown in the example honey-frying of Astragalus propinquus (Astragali Radix) root material.
\end{abstract}


Moreover, it may lead to the application of NADES in drug delivering systems. The different applications of honey in traditional medicine will be described, and its function and mechanism will be discussed. With the NADES concept we can now explain the various applications of honey as additive or adjuvant. As shown in numerous studies NADES are excellent solvents for medium polar bioactive compound. Besides improving the efficacy of herbal medicine, NADES also stabilize the active compounds. Honey has been used since ancient times for this purpose. The same effect can be achieved by a pure NADES, and the principle of improving solubility and stability can be applied to any poorly water-soluble medicine.

\section{Honeybee products, their ingredients and traditional applications}

\subsection{Traditional applications of honey}

Traditional Chinese medicine (TCM) utilizes numerous combinations of natural products. Most TCM products are prepared through extraction with water (e.g. making decoction). The limited number of extraction solvents raises a problem: not all compounds present in a single preparation will be fully extracted. To solve this problem, several natural materials, such as honey, wax, vegetable oil, and milk, are used as such or as adjuvant for preparing the final formulations. Of these materials, particularly honey has played an important role in traditional medicine for thousands of years. Traditionally it has been used as a medicine in itself, and as an additive, in the so-called Paozhi processing in which raw herbal drugs are cut into "decoction pieces" and treated with honey to improve their medical applications. Honey is also used as adjuvant for different types of pharmaceutical formulations, such as electuaries and honeyed pills. Hundreds of compounds have already been found in honeys from different sources. But the constituents present are greatly influenced by many factors, such as species of bees, species of plant sources, seasonal and geographical variation. Despite the large variation, the main components in all honeys are sugars such as glucose, fructose and sucrose. The normally solid sugar ingredients of honey can, in certain molar ratios, form natural deep eutectic solvent (NADES), explaining the liquid state of honey at room temperature. The physicochemical properties of such NADES may play an important role in preparing traditional medicines. Clarifying the role of honey in traditional medicines might help to rationalize traditional practices. Moreover it may lead to the application of NADES in drug delivering systems. Based on the applications of honey in traditional medicines, the potential further applications of honey 
and NADES will be discussed in this chapter. First of all, the different applications of honey in traditional medicine will be described, and its function will be discussed, including the perspectives of applications of NADES in drug delivering systems.

Honey is made by honeybees and some related insects from sugar containing plant secretions, e.g. flower nectar or honeydew from insects. Flower nectars are aqueous solutions mainly containing various sugars, such as sucrose, glucose and fructose. Honeybees make honey out of this, with glucose and fructose as the main ingredients along with about $18 \%$ of water. The brewing of honey involves two processes: the physical process reducing the water content from $40 \%$ to about $18 \%$ and the chemical process transforming di- and trisaccharides as well as polysaccharides (e.g. starch) into monosaccharides through invertases and amylases present in a hypopharyngeal gland secretion of honeybees (Yuan \& Feng, 2000). During these processes, honey matures to the point that the water content gets reduced to about $18 \%$. The honeybees store honey in the honeycombs sealed with beeswax for future consumption (Guo, 2004; Yu, 2017).

In addition to the sugars as main ingredients, there are other minor components in honey. Fresh honey is usually a transparent or translucent viscous liquid. During long-term storage at low-temperature and sometimes even at room temperature, part of honey sugars may crystallize. The color of honey can be nearly colorless to dark amber depending on the species of the source plants. The color generally comes from small amounts of pigments and minerals. Concerning fragrance, honey aroma is usually similar to that of the flowers, from which the nectar was collected. The aroma constituents, like esters, alcohols, phenols and acids are derived from the nectar but they are in part produced during the bees brewing process. The maturation of honey results in a unique aroma of honey. As the honey aroma compounds are volatile and sometimes labile substances, the composition of honey may undergo various changes during acid-base treatments, heating, or long-term storage, and the aroma may thus be lost in part (Guo, 2004; Yu, 2017).

Due to its unique sweetness, honey has been widely used in food since ancient times. It was used either as an additive or as a supplement for food processing. For example, honey is extensively used in various fields: sweeteners in confectioneries, brewing, pastries, and cooking. Also it is used as food additive such as: thickener, clarifier, stabilizer; humectant for beverages; and tobacco manufacturing (Guo, 2004).

Honey is also used in traditional medicines. In fact, recent studies reported a number of pharmacological effects for honey such as wound 
healing, pain relief, antibacterial activity, tissue regeneration, improving immunity, sedation, promoting digestion, hepatoprotection, protection of the cardiovascular system, and moistening lungs for relieving cough. Various biologically active compounds have been identified in honey. The antibacterial effect of honey might come from some of its constituents, from its high osmolarity in connection with the high concentration of sugars as well as the acidic character of honey (Guo, 2004; Hixon et al., 2019; Kurek-Górecka, Górecki, Rzepecka-Stojko, Balwierz, \& Stojko, 2020; Martinotti, Bucekova, Majtan, \& Ranzato, 2019; Nolan, Harrison, \& Cox, 2019).

Honeybeesmilk, so-called royal jelly, is another product made by the bees to feed the queen and infant worker bees. It is a milky or light yellow mixture secreted by the lingual gland and the mandibular gland of the head of young worker bees. It is based on the nutrients taken by the young worker bees after consumption of the brewed honey and naturally fermented pollen. It is slightly sweet, slightly sour, spicy and fragrant. Fresh royal jelly is reported to contain generally $64.5-68.6 \%(\mathrm{w} / \mathrm{w})$ of water and $12-14 \%$ $(\mathrm{w} / \mathrm{w})$ of protein. Dried royal jelly contains about $50 \%(\mathrm{w} / \mathrm{w})$ of protein, $15.6 \%(\mathrm{w} / \mathrm{w})$ of total lipids, and $0.8 \%$ of amino acids (Tang, Wang, \& Ren, 2005).

Propolis is a natural substance made by honeybees and used to protect the honeycomb. Propolis is an opaque sticky jelly which consists of a mixture of plant resins collected by bees and substances secreted by the bees' mandibular glands and wax glands (Commission of the Ministry of Health of the People's Republic of China, 2015). The color of propolis is brown, reddish brown, or grayish brown. Before spawning, it is applied to the honeycomb to seal small openings in the inner parts of the honeycombs, and to keep the honeycombs sterile. Thus, propolis keeps bees healthy as it is a natural broadspectrum antibiotic (Al-Waili, Al-Ghamdi, Ansari, Al-Attal, \& Salom, 2012). Propolis contains 50-60\% (w/w) of resin and balm, 30\% of beeswax, $10 \%(\mathrm{w} / \mathrm{w})$ of a volatile oil and pollen inclusions (Tang et al., 2005).

Beeswax (Cera Flava) is a substance secreted by four pairs of wax glands under the abdomen of two honeybees, Asian honeybee (Apis cerana Fabricius) or European one (A. mellifera Linnaeus) (Commission of the Ministry of Health of the People's Republic of China, 2015). Main components of beeswax are fatty acids and their esters with fatty alcohols. This mixture has good antiseptic and shaping properties (Tang et al., 2005). Beeswax is widely used in medicine, industry and agriculture. It can be used as an excipient for irritating drugs to reduce their degree of irritation. 
The character of low water-solubility of beeswax is being employed in drug delivery systems. A drug formulated in beeswax is slowly released via micropores or by slow dissolution of the beeswax. In TCM, beeswax is commonly used to make the shell of pills (Commission of the Ministry of Health of the People's Republic of China, 2015; Yang, 2012).

\subsection{Chemical ingredients of honey}

The many applications of honey fostered investigations on the ingredients of honey. These studies aimed at the identification of active ingredients and how this information could be used in quality control. The main components of honey are glucose and fructose, accounting for about $60-80 \%(\mathrm{w} / \mathrm{w})$, followed by sucrose (about 5\%,w/w), and organic acids $(0.1 \%, \mathrm{w} / \mathrm{w})$. The descriptions of international food standards (Codex Alimentarius Commission, 2011) and Chinese drugs standards for honey (Commission of the Ministry of Health of the People's Republic of China, 2015) are shown in Table 1. Officially honey should contain no less than $60 \%(\mathrm{w} / \mathrm{w})$ of fructose and glucose and no more than $5 \%(\mathrm{w} / \mathrm{w})$ sucrose. The water content should be less than $24 \%(\mathrm{w} / \mathrm{w})$. Minor sugars, like maltose, raffinose, kojibiose, melezitose, and lactose, have been also identified in honey, but their levels differ depending on source plants. The difference in chemical ingredients means variation of the physico-chemical properties of honey,

Table 1 International food standards, and Chinese drugs standards of honey.

\section{International food}

standards

Chinese drug standards

\begin{tabular}{lll}
\hline $\begin{array}{l}\text { Fructose/glucose/ } \\
(\mathrm{g} / 100 \mathrm{~g})\end{array}$ & $\begin{array}{l}\text { Common honey } \geq 60 \% \\
(\mathrm{w} / \mathrm{w})\end{array}$ & $\begin{array}{l}\geq 60 \%(\mathrm{w} / \mathrm{w}), \\
\text { fructose } / \mathrm{glucose} \geq 1.0\end{array}$ \\
\hline Sucrose & Common honey $\leq 5 \%$ & $\begin{array}{l}\text { Sucrose } \leq 5.0 \%(\mathrm{w} / \mathrm{w}), \\
\text { maltose } \leq 5.0 \%(\mathrm{w} / \mathrm{w})\end{array}$ \\
\hline $\begin{array}{ll}\text { Water content }(\%) \\
\begin{array}{l}\text { Acidity }(1 \mathrm{~mol} / \mathrm{L} \mathrm{NaOH}) / \\
(\mathrm{mL} / \mathrm{kg})\end{array}\end{array}$ & - & $\leq 24.0 \%$ \\
\hline
\end{tabular}

\begin{tabular}{ll}
\hline Conductivity & $\begin{array}{l}\text { Common } \\
\text { honey } \leq 0.8 \mathrm{~ms} / \mathrm{cm}\end{array}$ \\
\hline
\end{tabular}

\begin{tabular}{lll}
\hline Relative density & - & $\geq 1.349$ \\
\hline $\begin{array}{l}\text { Hydroxymethyl furfural } \\
(\mathrm{mg} / \mathrm{kg})\end{array}$ & - & 5 -Hydroxymethyl \\
\hline
\end{tabular}


like acidity, conductivity and relative density. Recent studies on the role of sugars in nature raise some interesting questions about the physicochemistry of honey (see below).

Honey is basically the mixture of components from plants, and bees as well as their fermented products. Therefore, besides the sugar ingredients, hundreds of other components have been identified in honeys. The chemical composition of honey is closely related to its source plants (Chen \& He, 2010; Guo, 2004; Hixon et al., 2019; Kurek-Górecka et al., 2020; Kuś, Jerković, Tuberoso, \& Šarolić, 2013; Mao, 2011; Martinotti et al., 2019; Nolan et al., 2019; Xia, Zhang, Wang, \& Ma, 2010). For example, the content of total flavonoids and phenolic acids was determined in five common honeys (jujube honey, prunella honey, acacia honey, vitex honey, rape flower honey) and the results showed that only jujube and prunella honey contained flavonoids. The highest content of total flavonoids found was $0.0015 \%(\mathrm{w} / \mathrm{w})$ while the total phenolic acids were found to be $0.050-0.063 \%(\mathrm{w} / \mathrm{w})$ in these five common honeys. The chemical variation in honeys may well cause a difference in biological activities. The characteristic chemicals of thyme- and chestnut honey are vitamins and polyphenolic acids, respectively, which reflect their antioxidant activity. In fact, compared with high antioxidant activity of chestnut honey, thyme honey does not have such high activity (León-Ruiz et al., 2013). The chemicals originating from source plants may cause not only beneficial effects, but also toxicity problems. For example, honey from Ericaceae family may contain toxic substances like grayanotoxin. The presence of pyrrolizidine alkaloids in honey became a social issue because of their liver toxicity, particularly for infants (Cramer \& Beuerle, 2012).

A broad range of other minor ingredients has been identified in honey: vitamins, amino acids, acids, choline, folate, volatiles, hormones, minerals, and proteins including enzymes (Kurek-Górecka et al., 2020; Martinotti et al., 2019; Nolan et al., 2019; Xia et al., 2010). Additionally, there are quite some organic acids in honey, and thus adding water results in weak acidity with $\mathrm{pH}$ of $3.2-4.5$ (about $10 \%$ of NADES in water).

\subsection{Honey as a NADES?}

Through our ${ }^{1} \mathrm{H}$ NMR-based metabolomics studies we found that many organisms and cells do contain large amounts of a few primary metabolites, often in similar molar concentrations, without any clear reason. We showed that certain combinations of solid primary metabolites, such as sugars, 
polyalcohols, amino acids, organic acids and bases, in certain molar ratios become a liquid at room temperature. These mixtures have a melting point usually far below that of the individual components, a phenomenon known as deep eutectic mixtures. The individual components in these mixtures have strong hydrogen bond acceptor or hydrogen bond donor characteristics and form a liquid with a supramolecular structure (Choi et al., 2011; Dai, Van Spronsen, Witkamp, Verpoorte, \& Choi, 2013). These natural deep eutectic solvents (NADES) can be divided into two groups: ionic liquids (ILs) in which a solid acid is mixed with a solid base, and deep eutectic solvents (DES) in which only neutral compounds (sugars, polyalcohols) are mixed, or a neutral compound is mixed with an amino acid, organic acid or base.

In our view the NADES represent a third liquid phase of intermediate polarity in living organisms (Choi et al., 2011). The occurrence of NADES in cells may explain the biosynthesis of poorly water- or fat-soluble compounds, like many secondary metabolites and macromolecules in plants. Also, in the survival of plants and other organism under cold, and drought conditions NADES might play a role, and in fact metabolomics studies show the presence of NADES ingredients in all kind of organisms under such stress conditions.

The occurrence of NADES in plants was proven by the presence of about equal molar concentrations of some sugars in the flower nectar, maple syrup (Choi et al., 2011). Bees feed on nectars that consist of NADES with different concentrations of water. Bees transform the nectar in to honey. Common honey consists of about $60-80 \%(\mathrm{w} / \mathrm{w})$ of glucose and fructose in about equal molar ratio with a small amount of sucrose (ca. 5\%, w/w) and about 20\% (w/w) water. According to the Chinese medicine standards the ratio of fructose/glucose in honey should be $\geq 1.0$. This ratio is consistent with the optimal NADES composition ratio for the mixture of fructose and glucose (Choi et al., 2011; Dai, Van Spronsen, et al., 2013). Honey can thus be considered to be a NADES containing up to about $20 \%$ of water, in which a plethora of plant compounds is dissolved. The role of nectar concerns three aspects. First of all as NADES are non-volatile, the nectar will remain as a liquid in the flower even under drought conditions. Second, poorly water-soluble volatile compounds that attract pollinators can be dissolved in a NADES like honey. Third, nectar is a major food source for pollinators like bees (Fig. 1).

In hunter-gathering period mankind fully relied on nature for gathering their food, and honey was probably the first sweetener. But they discovered 


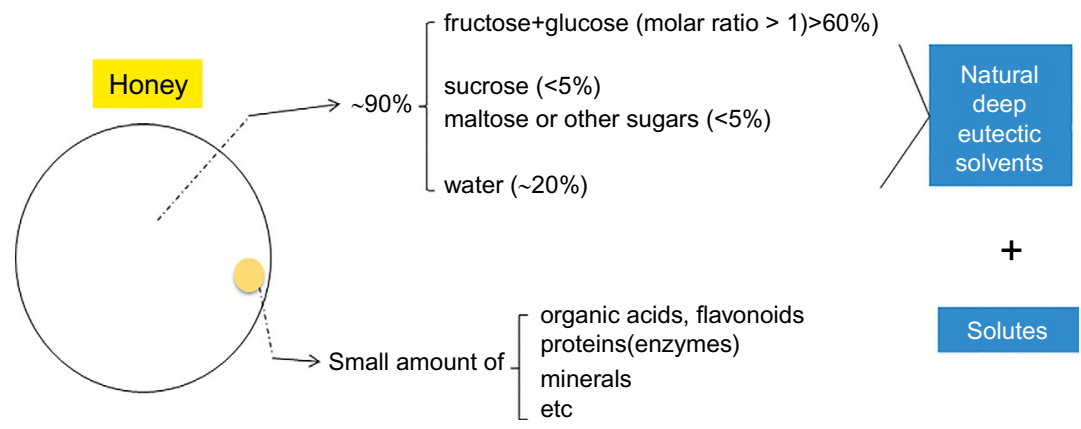

Fig. 1 Schematic diagram of honey composition.

also medical applications. A well-known and proven application is wound healing, which is related to its antibacterial activity (Hixon et al., 2019; Kurek-Górecka et al., 2020; Martinotti et al., 2019; Nolan et al., 2019). Along with its direct use as such, honey has been used as adjuvant in the preparation of various traditional medicines (Chen et al., 2018; Kuropatnicki, Klosek, \& Kucharzewski, 2018). To further elaborate the hypothesis that the uses of honey are associated with its NADES characteristics, we will discuss the role of honey in TCM in detail.

\section{Honey in traditional Chinese medicine}

Most preparations and formulations of TCM products include an extraction step. Though numerous bioactive metabolites with highly different physico-chemical properties are present in plant material and their formulations, extraction solvents are only a few: water, and water mixed with small amounts of ethanol. The most common TCM processing method is water-decoction, but to fully exploit the potential of active compounds, decoctions are not always the most suited method because of solubility issues. To deal with medium and low polar compounds diverse processing methods and formulations have been developed including pills, electuaries, syrups, various oils, tinctures, plasters, suppositories, glues, powders, granules, aerosols, etc. Honey has been applied in processing of various TCM since ancient times. The use of honey was already recorded in a Chinese document more than 2500 years ago. During the Tang Dynasty (AD 618-907) honey was reported to be a potential adjuvant for processing of medicinal plant materials, such as coating licorice with honey before 
processing. In the Classified Materia Medica written during the Song Dynasty (AD 960-1279) it was mentioned that "raw honey is good for treating eye diseases. Put honey in the eyes, and lie supine for half day." The Compendium of Materia Medica was written during the Ming Dynasty (AD 1368-1644) and described the biological functions of honey as clearing heat, nourishing the stomach and spleen, detoxifying, moistening dryness and relieving pain. In ancient times, it was recorded that honey should be kept beside well-water to keep it fresh for medical use.

The main functions of honey are described in the modern Chinese Pharmacopeia and are as follows: tonifying the stomach and spleen, moistening dryness, relieving pain, and detoxifying. For external use honey helps to regenerate tissues and heal wounds. It is also used for abdominal pain, dry cough caused by lung dryness, constipation caused by intestine dryness and detoxification of Aconite's toxins (e.g. aconitine analogs) in the bulbs of Aconitum species (Commission of the Ministry of Health of the People's Republic of China, 2015).

Honey, is also applied to the storage of various foods and medicines (Xie, 1987; Xiong, 2005). These applications of honey in TCM employ two types of honey: crude honey and refined honey. The crude naturally matured honey is used in applications for materials which are rich in nutrients and substances with therapeutic effects. Refined honey is mainly used as additive, for example, for honey stir-frying and as pharmaceutical adjuvant for making pills as well as electuaries. In the following sections, examples of applications of honey as adjuvant are discussed, based on the hypothesis that honey is a sugar-based NADES.

\subsection{Honey stir-fried preparations}

Paozhi is a treatment to improve the medical applications of herbal medicines in TCM. The functions of Paozhi treatment are altering the flavor, moderating the medicinal properties, and eliminating the side effects and detoxification of the crude drugs. The treatment encompasses such techniques as cutting, crushing, steaming, calcining, stir-frying, with or without adjuvants. Especially, honey is one of the most common adjuvants in the stir-frying of Paozhi.

Raw honey is usually refined by stir-frying under a gentle heat to reduce its water content down to $10-13 \%$, as well as to remove impurities, deactivate enzymes, kill microorganisms, and enhance adhesion (Yang, 2012). The refined honey, even with a low water content, could keep its liquidity, 
which is clear evidence for honey behaving like a NADES. The stir-frying with honey requires several steps: first the refined honey is diluted with boiling water (in a ratio of about $1 / 3$ of the weight of honey), this hot solution is added to the cleaned and cut plant materials, mixed thoroughly and the mixture is kept for a while to let the refined honey penetrate into the inner tissues of the plant material. After this preprocessing, the mixture of plant material and honey is stir-fried with gentle heat until the materials gain a specific color (Chinese Pharmacopeia), then the processed material is collected and cooled down. Generally, $25 \mathrm{~kg}$ refined honey is used for $100 \mathrm{~kg}$ plant material. The honey stir-fried pieces or slices are used to make a decoction with boiling water, the final formulation for the patient. As an example, morphological and metabolic change of the roots of Astragalus propinquus Schischkin caused by honey stir-fried process is shown in Fig. 2 (Dai et al., 2020).

According to TCM theory, the honey stir-frying of material of some medicinal plants have the following effects on the pharmacology:

1) moisten lungs for relieving cough, such as Stemona Radix (root of Stemona sessilifolia (Miq.) Miq.), Farfara flos (bud of Tussilago farfara L.), Asteris radix et rhizoma (root and rhizome of Aster tataricus L.f.);

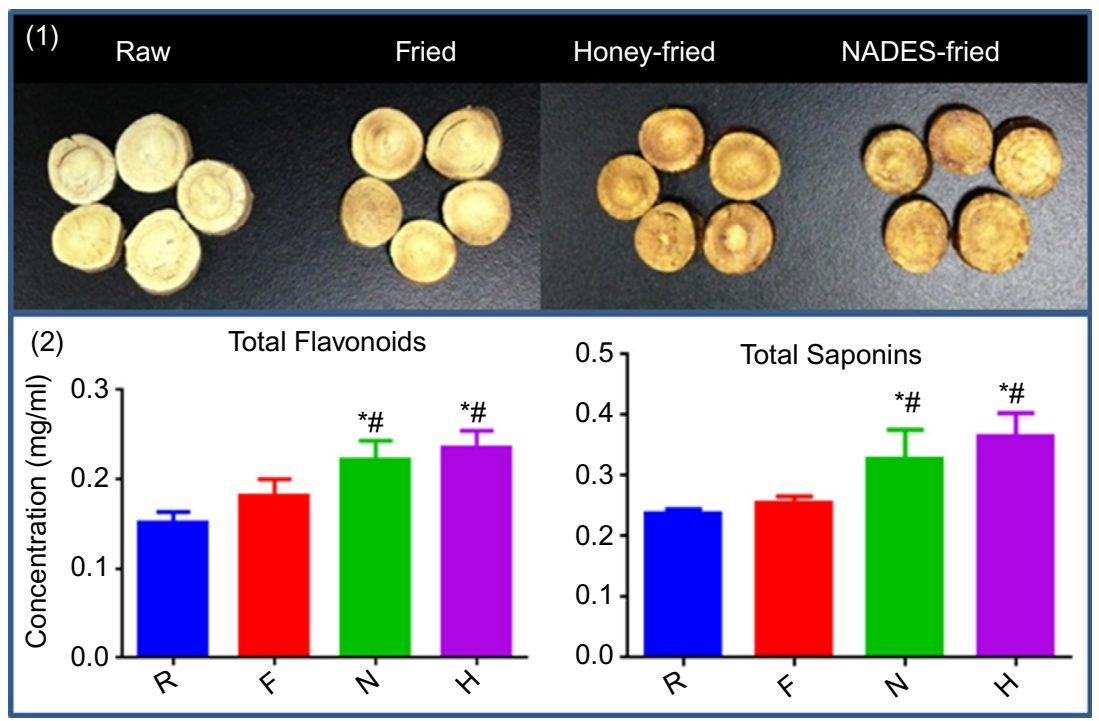

Fig. 2 Differently processed slices of Astragalus propinquus (Astragali Radix) and the level of total flavonoids and saponins in the decoctions estimated by UV spectroscopy. $\mathrm{R}$, raw material; F, fried material; N, NADES fried material; H, honey fried material $(n=6$, ${ }^{*} P<0.05$ vs $\mathrm{R}$ group; ${ }^{\#} P<0.05$ vs $\mathrm{F}$ group) (Dai et al., 2020). 
2) enhance the tonifying effects on spleen and nourishing $q i$, such as Astragali radix (root of Astragalus propinquus), Glycyrrhizae radix et rhizoma (root and rhizome of Glycyrrhiza uralensis Fisch.), Codonopsis radix (root of Codonopsis pilosula (Franch.) Nannf.);

3) moderate the properties of crude drugs. For example, Ephedra herba (aerial part of Ephedra sinica Stapf.) has a strong sweat driving force, which can be relieved by stir-frying with honey;

4) correct flavor and eliminate the side effects of drugs. For example, Aristolochia fructus (fruit of Aristolochia contorta Bunge) has a bitter taste, and is irritating to the stomach. Stir-frying with honey can enhance its antitussive effect, and it can also correct the bitter flavor to avoid vomiting (Jin, 1988).

What role does honey play in the stir-fry processing of plant materials? What components in honey play a major role? We hypothesized that the added honey penetrates the plant material and dissolves compounds present in the plant material during the processing. This may improve the solubilization of compounds in the final decoction step when the treated material is boiled with water. Moreover, the treatment may result in a more stable material to store, and may alter the chemical profile and enhance the biological activity of the final decoction products. This effect might be due to the major constituents of honey, the about equal molar amounts of glucose and fructose which form a NADES, known to readily solubilize medium polar compounds that hardly dissolve in water (Dai, Rozema, Verpoorte, \& Choi, 2016; Dai, Van Spronsen, et al., 2013; Dai, Verpoorte, \& Choi, 2014; Dai, Witkamp, Verpoorte, \& Choi, 2013). To prove this hypothetical mechanism, we studied the processing of Astragali Radix (AR) preparations as an example (Dai et al., 2020). Astragali Radix is a traditional medicine, which has been widely used since ancient times for relieving general weakness (Liu, Wang, \& Zhang, 2015). To prove a possible effect of a NADES the AR material was processed in different ways: Stir-frying without additives (samples F); stir-frying with honey (samples $\mathrm{H}$ ); and stir-frying with artificial honey made by mixing equal molar amounts of glucose and fructose (samples $\mathrm{N}$ ). Non-processed samples were used as control (Fig. 2). Decoctions were made of the processed AR samples following the method applied in the clinic. The decoction products were evaluated for their chemical ingredients and their bioactivity in some bioassays (Dai et al., 2020).

Total flavonoids and total saponins in the four AR decoctions were to some degree increased after all the processing when compared with the 
control samples. However, the level of isoflavonoids and saponins were far more increased by honey- and NADES-treated samples (the $\mathrm{H}$ and $\mathrm{N}$ decoctions) than in the decoction of the raw plant material (R) (Fig. 2). Apparently, the artificial honey using a sugar-based NADES, has the same effect as real honey, which supports the hypothesis that the effect of honey is similar as with a pure NADES in that it improves the solubility of medium polar plant secondary metabolites present in the plant material (Dai, Witkamp, et al., 2013).

The biological effects of these AR decoctions were tested on the endocrine-related estrogen receptor (ER)- $\alpha$, androgen receptor (AnRec), and antioxidant-related nuclear factor erythroid 2-related factor 2 (Nrf2) signaling pathways. No differences were observed between the $\mathrm{H}$ and $\mathrm{N}$ decoctions, both of which had significantly higher activities than the $\mathrm{R}$ and $\mathrm{F}$ decoctions (Fig. 3). Thus, it indicates that honey improved the biological effects of honey-stir-fried AR, which can be attributed to its NADES characteristics. The NADES increases the levels of total flavonoids and saponins in the $\mathrm{H}$ and $\mathrm{N}$ decoctions as measured by general methods for these types of compounds (Fig. 2) (Dai et al., 2020).

The chemical composition of the four decoctions was determined in detail using UPLC-Qtof-MS based metabolomics to explore the effects of honey. The levels of the aglycones calycosin ( $\mathrm{Ca}$ ) and formononetin (Fo) are relatively the highest in the non-processed samples (R), whereas the levels of their glucosides calycosin-7-O- $\beta$-D-glucoside $(\mathrm{CaG})$ and formononetin$7-O-\beta$-D-glucoside (FoG) were highest in the processed samples, and particularly in the $\mathrm{H}$ and the $\mathrm{N}$ group. The second notable difference was found in

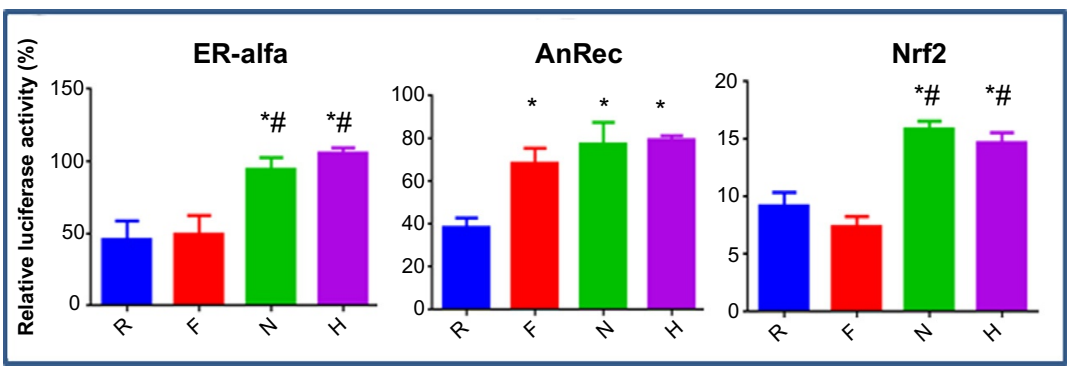

Fig. 3 The bioactivities of the AR decoctions (ER- $\alpha$, estrogen receptor- $\alpha$; AnRec, androgen receptor; Nrf2, nuclear factor erythroid-2-related factor; $n=6, * P<0.05$ vs R group; ${ }^{\#} P<0.05$ vs $F$ group). $R$, raw material; $F$, fried material; N, NADES fried material; $H$, honey fried material (Dai et al., 2020). 
the fried samples (F) group, where the calycosin-7-O- $\beta$-D-glucoside$6^{\prime \prime}-\mathrm{O}$-acetate (CaGA) and formononetin-7-O- $\beta$-D-glucoside- $6^{\prime \prime}-O$-acetate (FoGA) levels were approximately twofold higher than those in the control (R) samples, whereas the calycosin-7-O- $\beta$-D-glucoside- 6 "'-O-malonate (CaGM) and formononetin-7-O- $\beta$-D-glucoside- 6 "-O-malonate (FoGM) levels were slightly lower (not significant) in the $\mathrm{F}$ group than in the other three decoctions (Fig. 4). A significant increase in acetylastragaloside I (AgA I) relative level occurred in the fried samples (F) (Fig. 4), while the levels of astragaloside I (Ag I), isoastragaloside I (iAg I), and astragaloside II Ag II were lower in the $\mathrm{F}$ decoction than that in the control (R) (results not shown). The $\mathrm{H}$ and $\mathrm{N}$ samples also had slightly higher AGA I levels than the control, but not as much as in the only fried samples $(\mathrm{F})$. These results

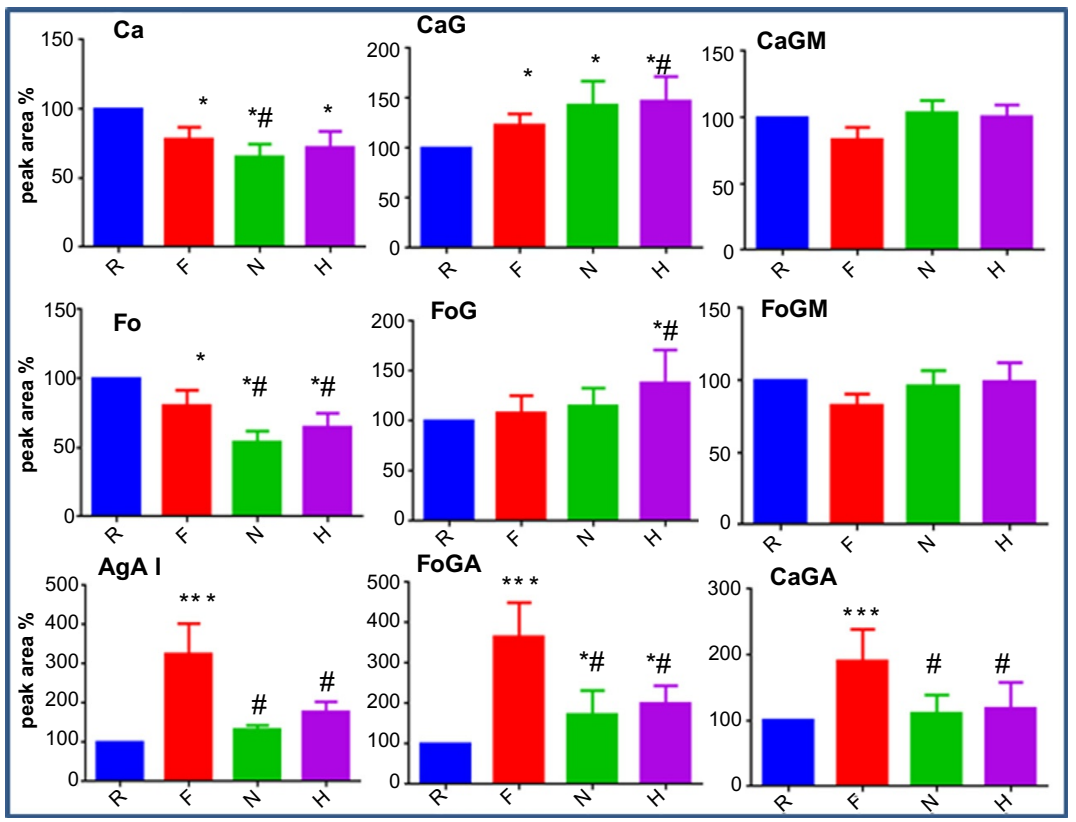

Fig. 4 Relative levels of some chemical constituents in the AR decoctions. $(n=12$, ${ }^{*} P<0.05$ vs $\mathrm{R}$ group; ${ }^{\#} P<0.05$ vs $\mathrm{F}$ group). Relative contents of components within fried samples were calculated with raw AR decoctions as $100 \%(\mathrm{Ca}$, calycosin; Fo, formononetin; $\mathrm{CaG}$, calycosin-7-O- $\beta$-D-glucoside; FoG, formononetin-7-O- $\beta$-D-glucoside; CaGM, calycosin-7-O- $\beta$-D-glucoside- 6 "-O-malonate; FoGM, formononetin-7-O- $\beta$-Dglucoside- 6 "-O-malonate; CaGA, calycosin-7-O- $\beta$-D-glucoside- $6^{\prime \prime}-O$-acetate; FoGA, formononetin-7-O- $\beta$-D-glucoside- 6 "-O-acetate; AgA I, acetylastragaloside I) (R, raw material; F, fried material; N, NADES fried material; $H$, honey fried material) (Dai et al., 2020). 
show that the stir-frying does affect the metabolic profile of the formulations. This is in line with a change in the biological activities.

Several explanations might explain the changes in the metabolic profiles.

The NADES in the honey stir-frying might (Ma, Wei, Wang, \& Hattori, 2009; Zheng, Shi, Jie, \& Lu, 2014):

- promote the conversion of aglucones to glucosides;

- inhibit the hydrolysis of the glucosides;

- inhibit acetylation of isoflavonoids and astragalosides;

- increase deacetylation of acetyl-containing isoflavonoids and astragalosides;

- inhibit the hydrolysis of the malonylated and/or acetylated glucosides;

- improve the extraction of the isoflavonoids glucosides if compared to the aglycones.

It thus seems that the stir-frying is an important step for the quality of the traditional medicine, so further research is required to study this effect in more detail.

A protective effect of honey might come from the presence of a large amount of reducing sugars, which can prevent the transformations of labile ingredients of the medicinal materials. Such a NADES protective effect is supported by a previous study in which a stabilizing effect of NADES was observed for flavonoids during extraction and storage (Dai et al., 2016). The CaGM might be transformed during frying into an acetyl-containing molecule (CaGA), i.e. a transesterification (Lin et al., 2000; Ma et al., 2009).

The effect of honey on the absorption of active compounds was determined in rats with CG as example. Calycosin-7-O- $\beta$-D-glucoside is an important flavonoid in AR with the ER- $\alpha$, AnRec and Nrf2 activities. The bioavailability of $\mathrm{CaG}$ was markedly increased with honey or NADES-treated samples, based on the plasma concentration and permanence period in rats (Fig. 5). A similar bioavailability improving effect of NADES

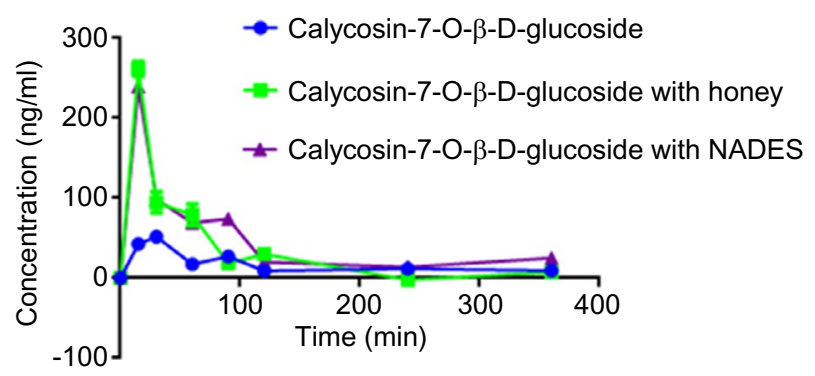

Fig. 5 Time course (mean \pm SD) of plasma concentration of calycosin-7-O- $\beta$-D-glucoside in SD rats following oral administration of calycosin-7-O- $\beta$-D-glucoside $(20 \mathrm{mg} / \mathrm{kg})$ with or without honey, or honey analog (NADES) (Dai et al., 2020). 
was reported for rutin, a flavonoid diglycoside (Faggian et al., 2016). The present study provides evidence for a synergistic effect of honey with active compounds in improving activity of AR. This result implies that the NADES structure of honey plays a major role in improving the bioavailability of CG. Apparently the honey- and NADES stir-frying process modify and increase the pharmacological activities of the drug in a favorable way.

\subsection{Traditional formulations: Honeyed pills and other dosage forms}

In addition to the use as an adjuvant in processing medicinal plants honey is also often used for pharmaceutical purposes in TCM. Especially, honeyed pills and electuaries are quite common formulations. In the production of honeyed pills, the quality of honey is strictly controlled; not only for the chemical ingredients (like water, enzymes, microorganism, and minor impurities) but also for its physical properties. According to the degree of refining, refined honey is divided into three categories: tender honey, medium honey, and old/hard honey. Each of them has a specific viscosity and water content, which eventually decides their specific applications. For example, tender honey with a water content of $17-20 \%$, and a relative density of about $1.35 \mathrm{~g} \mathrm{~cm}^{-3}$, is suitable for binding fine sticky powdered crude plant materials; the more viscous medium honey with $14-16 \%$ water and about $1.37 \mathrm{~g} \mathrm{~cm}^{-3}$ relative density is used to bind fine powders of loose crude plant materials; the most viscous old honey with $10 \%$ water content and about $1.40 \mathrm{~g} \mathrm{~cm}^{-3}$ relative density is applied for minerals and fibrous fine powders of plant materials.

The preparation of honeyed pills can be divided into several steps: cutting and grinding of the plant material into a fine powder; mixing the powder and honey (about 1:1 to 1:1.5) into a roll with proper hardness and plasticity, which then is cut and made into pills (Fig. 6) (Yang, 2012).

There are also other types of pills derived from honeyed pills (Table 2). In order to reduce the size of honey pills, the powdered plant materials used in honey pills is (partially) replaced by their extracts in so-called concentrated pills. According to the used adjuvants, the concentrated pills can be divided into concentrated water pill, concentrated honeyed pill, and water-honeyed pill. Although concentrated water pills are currently the most common ones, still the other types of honey added pills have some specific applications. Water-honeyed pills are made from a fine powder of the plant material and diluted refined honey as adhesive. Compared with honeyed pills, they save honey, reduce costs and are easier to store. Wax pills are made from a fine powder of the plant material mixed with beeswax as adhesive. Beeswax 


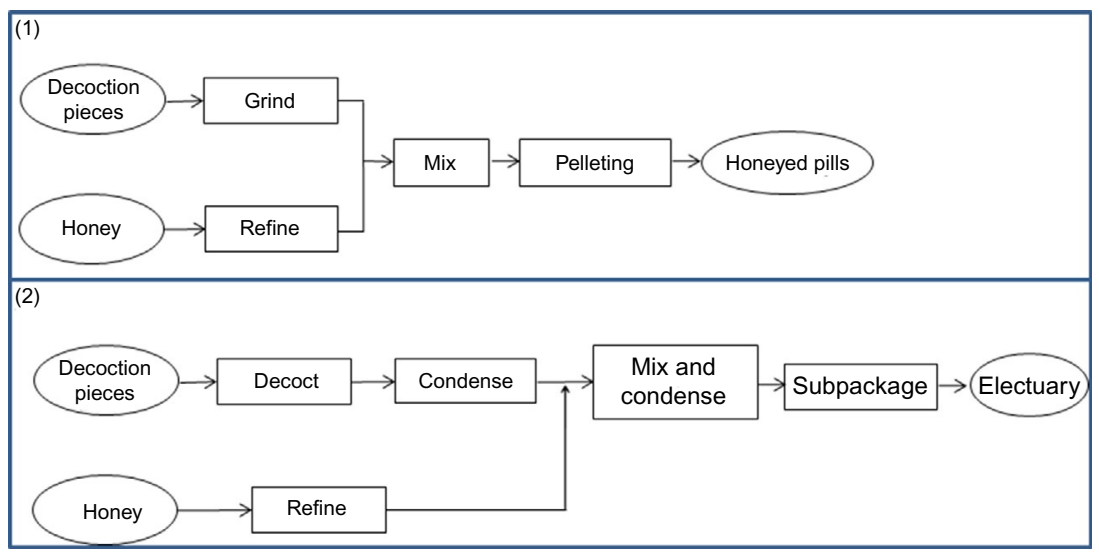

Fig. 6 Typical steps in the preparation of honeyed pills (1) and electuaries (2) (Yang, 2012).

Table 2 Characteristics and applications of different dosage forms with honey as adjuvant/ingredient.

\begin{tabular}{llll} 
Usage form & Advantage & Disadvantage & Applications \\
\hline Electuary & $\begin{array}{l}\text { High drug concentration, } \\
\text { easy to store and } \\
\text { convenient to take }\end{array}$ & $\begin{array}{l}\text { Not suitable } \\
\text { for heat- } \\
\text { sensitive or } \\
\text { volatile drugs }\end{array}$ & $\begin{array}{l}\text { Suitable for chronic } \\
\text { diseases }\end{array}$ \\
\hline $\begin{array}{l}\text { Honeyed pills } \\
\text { Slow release in the } \\
\text { gastrointestinal tract, long- }\end{array}$ & Large in size & $\begin{array}{l}\text { Suitable for antitussive } \\
\text { and expectorant drugs, } \\
\text { lasting effect }\end{array}$ & $\begin{array}{l}\text { and drugs for tonifying } \\
\text { spleen-stomach }\end{array}$ \\
\hline $\begin{array}{l}\text { Concentrated } \\
\text { pill }\end{array}$ & $\begin{array}{l}\text { Small in size, easy to carry } \\
\text { and store }\end{array}$ & $\begin{array}{l}\text { Long heating } \\
\text { time }\end{array}$ & $\begin{array}{l}\text { Not suitable for heat- } \\
\text { unstable drugs }\end{array}$ \\
\hline $\begin{array}{l}\text { Water- } \\
\text { honeyed pill }\end{array}$ & $\begin{array}{l}\text { Save honey, reduce costs, } \\
\text { easy to store }\end{array}$ & $\begin{array}{l}\text { Long heating } \\
\text { time }\end{array}$ & $\begin{array}{l}\text { Not suitable for heat- } \\
\text { unstable drugs }\end{array}$ \\
\hline Wax pill & $\begin{array}{l}\text { Prolonged efficacy, } \\
\text { prevent poisoning or } \\
\text { irritation of drug in } \\
\text { gastrointestinal tract }\end{array}$ & $\begin{array}{l}\text { Unable to } \\
\text { control the } \\
\text { rate of drug } \\
\text { release }\end{array}$ & $\begin{array}{l}\text { Suitable for toxic or } \\
\text { irritative drugs }\end{array}$ \\
\hline
\end{tabular}

is an interesting material, not only for formulation but also as drug delivery system. Beeswax is poorly water soluble, neither in vivo, nor in vitro. The application of beeswax significantly prolongs the pharmacological effects of a drug because of the slow release, and prevents irritation of the 
gastrointestinal tract by the drug and may avoid possible toxic effects (Commission of the Ministry of Health of the People's Republic of China, 2015; Yang, 2012).

Another common dosage form of TCM with honey as additive is an electuary. An electuary is a semi-liquid preparation made by boiling plant materials with water, concentrating the decoction, and adding refined honey or sugar (invert sugar). Electuaries are mostly used for the treatment of some chronic diseases, with the advantages of a high drug concentration, easy production and storage. Due to the boiling process, however, it is recommended that plant material containing heat-sensitive or volatile compounds should not be made into electuaries. The preparation of an electuary consists of several steps: decoction with water; concentration of the extract; mixing with refined honey; paste collecting; and subpackaging (Fig. 6). When the density of the concentrated extract reaches around at $1.21-1.25 \mathrm{~g} \mathrm{~cm}^{-3}$ at $80^{\circ} \mathrm{C}$, refined honey is added. Generally, the amount of refined honey or sugar added does not exceed three times of the amount of paste. The paste is continuously stirred and heated until a general relative density of paste reaches about $1.4 \mathrm{~g} \mathrm{~cm}^{-3}$. Then the paste should be divided into clean and dry containers.

As pharmaceutical adjuvant, viscous liquid or semi-liquid honey is mostly used to make pills. For this application, honey with higher content of fructose than glucose is required. This kind of honey can not only increase the moisture of honeyed pills, but also take advantage of the sweetness of fructose. Refining process makes honey more viscous and with lower water level, that brings the advantages of strong adhesive and plasticity characteristics as an adjuvant. Also, refined honey can give a special character to the pills, e.g. round shapes, moisture, and softness.

The use of honey in the preparation of traditional medicines, can be explained by the NADES character of honey. The typical characteristics of NADES are non-volatile, liquid in a very broad temperature range, high viscosity, easy to mix with water, antimicrobial activity, good solubility of medium polar poorly water-soluble compounds, and increased stability of phenolic compounds (Dai et al., 2014, 2016, 2020; Dai, Van Spronsen, et al., 2013; Dai, Witkamp, et al., 2013; Dai, Witkamp, Verpoorte, \& Choi, 2015). The honeyed pills are slow to disintegrate in the digestive tract, which enables a long-lasting effect. Also, there might be an effect on the absorption of ingredients of TCM. The sustained release effect of honey pills on the bioavailability of some important active and toxic components has been observed in the case of Aconitum roots when comparing honey pills and water pills (Gan et al., 2018). 


\section{The physico-chemical properties of honey as NADES}

Above we discussed the roles of honey in Chinese medicine. The observations made in various studies clearly show that honey has the typical characteristics of a NADES and the studies on stir-fried Astragalis Radix with artificial honey showed similar results as the natural honey. Here we will discuss in some more detail the properties of NADES, and specifically the sugar-based NADES, like honey.

\subsection{Hygroscopicity}

When the air is humid, honey can absorb water from the air. The absorption capacity of honey, i.e. the hygroscopicity, depends on the percentage of water already present in honey, and the water vapor pressure in the air. When the air is dry, honey evaporates water.

\subsection{Viscosity}

The main factors that determine the viscosity of honey are water content and temperature. The lower the water content, the higher the viscosity. The viscosity decreases with increasing temperature (Dai et al., 2015; Guo, 2004).

\subsection{Refractive index}

Determination of the refractive index of honey is a simple and accurate method to identify the water content of honey. The refractive index of honey decreases with the increase of water content and temperature (Guo, 2004).

\subsection{Optical rotation}

Optical rotation is related to its structure, water content, and other factors. But the optical rotation is mainly due to the sugars in honey, in which glucose and sucrose are dextrorotary, and fructose is levorotary. Normally mature honey is levorotatory. If glucose or sucrose is added, it will cause a change of rotation, and the levorotation will be smaller. Therefore, the rotation can be used to confirm the authenticity of honey (Guo, 2004).

The physical properties of honey are consistent with the reported physical data of artificial honey, a glucose-fructose based NADES (Dai et al., 2015; Dai, Van Spronsen, et al., 2013). It means that the natural deep eutectic solvent's part of honey determines the main physico-chemical properties of honey. 


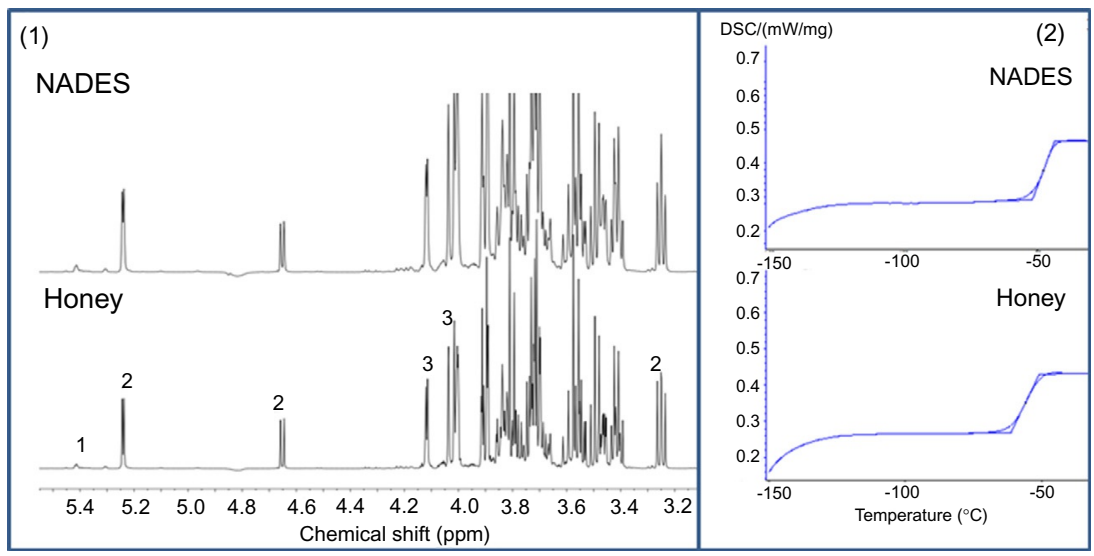

Fig. 7 The ${ }^{1} \mathrm{H}$ NMR spectra (A) and DSC curve (B) of honey and man-made honey, namely, natural deep eutectic solvents (NADES). The honey and NADES are composed of a mixture of glucose, fructose, and sucrose with certain amount of water (1: sucrose; 2: glucose; 3: fructose) (Dai et al., 2020).

To test the hypothesis, that honey might be a NADES, some sugar-based NADES that mimic honey were investigated for their physico-chemical characteristics using several methods. The ${ }^{1} \mathrm{H}$ NMR spectra showed that the NADES and honey have a similar chemical composition and the similar chemical shifts which support the occurrence of strong hydrogen bonding between the constituents, creating a supramolecular structure (Fig. 7). The differential scanning calorimetry (DSC) test showed a glass transition point (from -50 to $-60^{\circ} \mathrm{C}$ ) but no melting point of NADES and honey, which suggests that the NADES and honey have similar supramolecular structures.

Recently the presence of active macromolecules in honey was postulated (Brudzynski et al., 2017). A supramolecular structure of honey was proposed because the UV spectra of honey change upon dilution with water. The changes were thought to be due to light scattering particles present in honey. These particles were supposed to be formed by self-assembly from macromolecules present in honey. By means of scanning electron microscopy it seemed like two phases were present: dense globules in a sugar solution. Dilution with water resulted at a certain point in the microparticles falling apart in nanoparticles. Below this threshold level honey lost its antibacterial activity. Do these observations contradict the NADES hypothesis? Most honeys are rich in phenolic compounds, like flavonoids, anthocyanins and cinnamic acid derivatives which have a low to medium solubility in 
water, but dissolve well in NADES. These compounds are very sensitive for shifts in their UV spectra, among others, the $\mathrm{pH}$, and metals cause shifts in the UV spectra. In honey itself there is no $\mathrm{pH}$, as by definition the $\mathrm{pH}$ is the concentration of protons in water, but dilution of honey with water changes the UV spectra of the phenolics due to the effect of the acids present in honey. Supramolecular structures of NADES were clearly proven by ${ }^{1}$ H NMR spectroscopy (Choi et al., 2011; Dai et al., 2015), showing that the NADES components and water are strongly interacting through H-bonding. Upon dilution the signals of the components in the ${ }^{1} \mathrm{H}$ NMR start to shift. Below about 50\% NADES in water, the ${ }^{1} \mathrm{H}$ NMR spectra do not change anymore and they look like an aqueous solution of the NADES components (Dai et al., 2015). The gradual shift of the proton resonances of the sugars upon dilution can be explained by a fast equilibrium between two phases, one in which the NADES components are organized in small molecular aggregates, and in part are freely moving in the water. The changes in the chemical shifts of the protons in the ${ }^{1}$ HNMR spectra reflect the time the protons are in one or the other phase. The self-assembly in honey observed by Brudzynski et al. (2017) may involve the sugar molecules that interact with the poorly water-soluble phenolics like flavonoids and anthocyanins (Dai et al., 2014). It is well known that mixtures of anthocyanins, flavones and cinnamic acid derivatives may form complexes, and in a number of plant species various anthocyanic vacuolar inclusions (AVI) have been observed that contain such phenolic compounds. AVI with different structures have been found, often without a membrane, and some types seemed to have a protein matrix (Andersen \& Jordheim, 2010). The assumption that active macromolecules are responsible for the formation of colloidal particles in honey is in line with this (Brudzynski et al., 2017). The formation of particles in honey also fits the hypothesis of Choi et al. (2011) that NADES function as a third phase in cells that can dissolve poorly water-soluble phenolics and form some sort of vesicles in which the biosynthesis can occur (e.g. in the ER). Also transport of these compounds to the vacuole could be through these particles that contain the NADES in which the phenolic compounds are dissolved. Further research is needed to integrate the physico-chemical properties of honey with the biological system.

\section{Other adjuvants in TCM}

Sugars in honey might be the physico-chemical platform for the functions of honey, like to foster or support the roles of minor bioactive 
metabolites. The NADES may also be formed during the processing from compounds in the herbal medicine and added compounds, like sugars, sugar alcohols, amino acids, organic bases and acids and ethanol. Some common adjuvants applied in processing of TCM are rice wine, vinegar, salt, and oil.

Rice wine is used in preparing pharmaceutical formulations. Rice wine is brewed from sticky rice and contains kojic acid. It is generally a yellow transparent liquid with a mellow fragrance. Stir-frying with rice wine, helps to dissolve the active compounds and thus increases the efficacy of the drug. Drugs stir-fried with rice wine include Scutellaria radix (roots of Scutellaria baicalensis Georgi), Rhei radix et rhizoma (rhizomes of Rheum palmatum L.), Paeoniae radix alba (roots of Paeonia lactiflora Pall.), Angelicae sinensis radix (roots of Angelica sinensis (Oliv.) Diels), etc.

Vinegar for pharmaceutical use should be food grade vinegar, and the longer it is stored, the better, yielding the so-called "mature vinegar." Vinegar is produced from oxidation of alcohol in fermented rice, wheat, sorghum or other grains. Vinegar is acidic and can dissolve free alkaloids in water by protonation, and thus improving the therapeutic effect. The traditional medicines that often are stir-fried with vinegar are Phytolaccae radix (roots of Phytolacca acinosa Roxb.), Bupleuri Radix (roots of Bupleurum chinense DC.), and Cyperi rhizoma (rhizomes of Cyperus rotundus L.).

Baking herbal medicines with salt water is a common procedure for Morindae officinalis radix (roots of Morinda officinalis F.C. How), Foeniculi fructus (fruits of Foeniculum vulgare Mill.), and Plantaginis semen (seeds of Plantago asiatica L.).

\section{Prospects}

Nature is a treasure house for bioactive compounds, but also for adjuvants that can be used in formulating medicines. Honey is a clear example. Although the individual character of a honey is governed by its minor plant-originating metabolites, all types of honey share two common metabolites: glucose and fructose in about equal molar amounts, representing $60-80 \%$ of the weight of honey. According to our hypothesis, in Nature the combination of various sugars and other abundant primary metabolites in certain molar ratios will form NADES. This can be on subcellular level but also on the level of a tissue or in plant saps like nectar. This hypothesis generates many ideas for possible applications. In fact our ancestors already discovered the use of honey to improve traditional medicines. With the NADES concept we can now explain the various applications of honey as additive or adjuvant. As shown in numerous studies 
NADES are excellent solvents for medium polar bioactive compound. Besides improving the efficacy of herbal medicine, NADES also stabilize the active compounds. Honey has been used since ancient times for this purpose. The same effect can be achieved by a pure NADES, and the principle of improving solubility and stability can be applied to any poorly water-soluble medicine.

\section{References}

Al-Waili, N., Al-Ghamdi, A., Ansari, M. J., Al-Attal, Y., \& Salom, K. (2012). Synergistic effects of honey and propolis toward drug multi-resistant Staphylococcus aureus, Escherichia coli and Candida albicans isolates in single and polymicrobial cultures. International Journal of Medical Sciences, 9, 793-800.

Andersen, O. M., \& Jordheim, M. (2010). Overview and introduction. In L. Mander, \& H. W. Liu (Eds.), Comprehensive natural products II chemistry and biology (pp. 547-614). Oxford: Elsevier Ltd.

Brudzynski, K., Miotto, D., Kim, L., Sjaarda, C., Maldonado-Alvarez, L., \& Fuk, H. (2017). Active macromolecules of honey form colloidal particles essential for honey antibacterial activity and hydrogen peroxide production. Scientific Reports, 7, 7637. https://doi.org/10. 1038/s41598-017-08072-0.

Chen, C. G., \& He, S. Y. (2010). Bee products. Beijing: China Agricultural Press.

Chen, L. L., Verpoorte, R., Yen, H. R., Peng, W. H., Cheng, Y. C., Chao, J., et al. (2018). Effects of processing adjuvants on traditional Chinese herbs. Journal of Food and Drugs Analysis, 26, S96-S114.

Choi, Y. H., van Spronsen, J., Dai, Y., Verberne, M., Hollmann, F., Arends, I. W., et al. (2011). Are natural deep eutectic solvents the missing link in understanding cellular metabolism and physiology? Plant Physiology, 156, 1701-1705.

Codex Alimentarius Commission, 2011. (Adopted in 1981. Revisions 1987 and 2001). CODEX STAN 12-1981 codex standard for honey.

Commission of the Ministry of Health of the People's Republic of China. (2015). Pharmacopoeia of the people's republic of China. Vol. I. Beijing: Ministry of Public Health of the People's Republic of China.

Cramer, L., \& Beuerle, T. (2012). Detection and quantification of pyrrolizidine alkaloids in antibacterial medical honeys. Planta Medica, 78, 1976-1982.

Dai, Y., Jin, R., Verpoorte, R., Lam, W., Cheng, Y. C., Xiao, Y., et al. (2020). Natural deep eutectic characteristics of honey improve the bioactivity and safety of traditional medicines. Journal of Ethnopharmacology, 250, 112460. https://doi.org/10.1016/j.jep. 2019.112460.

Dai, Y., Rozema, E., Verpoorte, R., \& Choi, Y. H. (2016). Application of natural deep eutectic solvents to the extraction of anthocyanins from Catharanthus roseus with high extractability and stability replacing conventional organic solvents. Journal of Chromatography A, 1434, 50-56.

Dai, Y., Van Spronsen, J., Witkamp, G. J., Verpoorte, R., \& Choi, Y. H. (2013). Natural deep eutectic solvents as new potential media for green technology. Analytica Chimica Acta, 766, 61-68.

Dai, Y., Verpoorte, R., \& Choi, Y. H. (2014). Natural deep eutectic solvents providing enhanced stability of natural colorants from safflower (Carthamus tinctorius). Food Chemistry, 159, 116-121. 
Dai, Y., Witkamp, G. J., Verpoorte, R., \& Choi, Y. H. (2013). Natural deep eutectic solvents as new extraction media for phenolic metabolites in Safflower. Analytical Chemistry, 85, $6272-6278$.

Dai, Y., Witkamp, G. J., Verpoorte, R., \& Choi, Y. H. (2015). Tailoring properties of natural deep eutectic solvents with water to facilitate their applications. Food Chemistry, 187, 14-19.

Faggian, M., Sut, S., Perissutti, B., Baldan, V., Grabnar, I., \& Dall'Acqua, S. (2016). Natural deep eutectic solvents (NADES) as a tool for bioavailability improvement: Pharmacokinetics of rutin dissolved in proline/glycine after oral administration in rats: Possible application in nutraceuticals. Molecules, 21, 1531.

Gan, J., Wang, C., Song, Z., Liu, Y., Ning, Z., Ma, X., et al. (2018). Effect of refined honey on pharmacokinetics of 9 different alkanoids in Fuzi Lizhongwan. Chinese Journal of Experimental Traditional Medical Formulae., 24, 90-96.

Guo, F. B. (2004). Honey and its magical effects. Beijing: China Agricultural Press.

Hixon, K. R., Klein, R. C., Eberlin, C. T., Linder, H. R., Ona, W. J., \& Gonzalez, H. (2019). A critical review and perspective of honey in tissue engineering and clinical wound healing. Advances in Wound Care, 8, 403-415.

Jin, S. Y. (1988). Science of processing Chinese materia medica. Jiangsu: Jiangsu Science and Technology Press.

Kurek-Górecka, A., Górecki, M., Rzepecka-Stojko, A., Balwierz, R., \& Stojko, J. (2020). Bee products in dermatology and skin care. Molecules, 25, 556.

Kuropatnicki, A. K., Klosek, M., \& Kucharzewski, M. (2018). Honey as medicine: Historical perspectives. Journal of Apicultural Research, 57, 113-118.

Kuś, P. M., Jerković, I., Tuberoso, C. I., \& Šarolić, M. (2013). The volatile profiles of a rare apple (Malus domestica Borkh.) honey: Shikimic acid-pathway derivatives, terpenes, and others. Chemistry \& Biodiversity, 10, 1638-1652.

León-Ruiz, V., González-Porto, A. V., Al-Habsi, N., Vera, S., San Andrés, M. P., \& Jauregi, P. (2013). Antioxidant, antibacterial and ace-inhibitory activity of four monofloral honeys in relation to their chemical composition. Food \& Function, 4, 1617-1624.

Lin, L. Z., He, X. G., Lindenmaier, M., Nolan, G., Yang, J., Cleary, M., et al. (2000). Liquid chromatography-electrospray ionization mass spectrometry study of the flavonoids of the roots of Astragalus mongholicus and A. membranaceus. Journal of Chromatography $A$, 876, 87-95.

Liu, Y. Z., Wang, Z. M., \& Zhang, J. Z. (2015). Dietary Chinese herbs. Springer Vienna.

Ma, C. M., Wei, Y., Wang, Z. G., \& Hattori, M. (2009). Triterpenes from Cynomorium songaricium-Analysis of $\mathrm{HCV}$ protease inhibitory activity, quantification, and content change under the influence of heating. Journal of Natural Medicine, 63, 9-14.

Mao, W. L. (2011). Study on the components of propolis and honey. Thesis Henan: Henan University.

Martinotti, S., Bucekova, M., Majtan, J., \& Ranzato, E. (2019). Honey: An effective regenerative medicine product in wound management. Current Medical Chemistry, 26, $5230-5240$.

Nolan, V. C., Harrison, J., \& Cox, J. (2019). Dissecting the antimicrobial composition of honey. Antibiotics, 8, 251.

Tang, D. G., Wang, Y. X., \& Ren, X. Y. (2005). Honey. Beijing: Beijing Science and Technology Press.

Xia, L. Y., Zhang, X. Y., Wang, T. X., \& Ma, Y. S. (2010). Study on the chromatographic fingerprint of volatile constituents from acacia honey. Agricultural Science \& Technology, $11,42-44$.

Xie, G. Y. (1987). Honey and traditional Chinese medicine. Bee Journal, 02, 31-32. 
Xiong, P. H. (2005). Application of honey in processing and preparation of traditional Chinese medicine. Li Shizhen Medicine and Materia Medica Research, 16, 35-36.

Yang, M. (2012). Traditional Chinese medicine pharmaceutics. Beijing: China Traditional Chinese Medicine Press.

Yu, Z. H. (2017). Study on the changes of compositions in honey during maturing. Thesis Fujian: Fujian Agricultural and Forestry University.

Yuan, Z. L., \& Feng, F. (2000). Bee product processing technology and health care. Beijing: Scientific and Technical Documents Publishing Press.

Zheng, N., Shi, Y. L., Jie, Y. X., \& Lu, W. (2014). Stability and transformation of astragaloside IV, III and I. Central South Pharmacy, 12, 1062. 